\title{
Electron-Ion Temperature Equilibration at Collisionless Shocks in Supernova Remnants
}

\author{
Cara E. Rakowski \\ Harvard-Smithsonian Center for Astrophysics, 60 Garden St. MS-70, Cambridge, \\ MA 02138, USA
}

\begin{abstract}
The topic of this review is the current state of our knowledge about the degree of initial equilibration between electrons, protons and ions at supernova remnant (SNR) shocks. Specifically, the question has been raised as to whether there is an inverse relationship between the shock velocity and the equilibration similar to the relationship between equilibration and Alfvén Mach number seen in interplanetary shocks (Schwartz et al., 1988). This review aims to compile every method that has been used to measure the equilibration and every SNR on which they have been tested. I review each method, its problems and uncertainties and how those would effect the degree of equilibration (or velocity) inferred. The final compilation of observed electron to proton temperature ratios as a function of shock velocity gives an accurate, conservative picture of the state of our knowledge and the avenues we need to pursue to make progress in our understanding of the relation between the velocity of a shock and the degree of equilibration.
\end{abstract}

Key words:

shock waves, supernova remnants, cosmic rays, X-rays: ISM, ISM: individual (Cygnus Loop, RCW 86, DEM L71, Tycho's supernova remnant, SN 1006, 1E 0102.2-7219), supernovae: individual (SN 1987A)

\section{Introduction}

The nature of electron and ion heating behind collisionless shocks remains an open question in shock physics. While the overall kinetics of a shock can be described simply in terms of the Rankine-Hugoniot solutions to the equations for conservation and continuity (see e.g. McKee and Hollenbach, 1980), the

Email address: crakowski@cfa.harvard.edu (Cara E. Rakowski). 
fraction of the shock's kinetic energy that is transferred to the thermal and cosmic-ray populations of electrons and ions is unknown. Theoretically, anything between $\left(T_{e} / T_{p}\right)_{0} \sim m_{e} / m_{p}$ to rapid full equilibration has been proposed. However mounting observational evidence points to an intermediate degree of initial electron heating, where the disparity between electron and ion heating increases with shock velocity. This review examines these measurements in order to point the way towards further progress.

Modeling non-linear acceleration of particles in high Mach number collisionless shocks remains a challenge. There are numerous analytically derived instabilities that could be important, but it is not necessarily straight-forward to interpret particle simulations in terms of these processes. While comparing the simulations to observations of solar wind shocks has been quite successful, the results may not have a strong bearing on the important processes in supernova remnant (SNR) shocks which are significantly faster and less dense. For a recent, thorough review of the open problems in collisionless-shock physics see Lembege et al. (2004). For the purpose of this review, it is important to keep in mind that the electro-magnetic waves that define these instabilities and the shock itself will affect the ions and electrons differently due to the large inertial difference.

Given the current ambiguity regarding the partitioning of energy at a shock, interpretations of SNR observations commonly assume purely thermal particle distributions and consider the extreme cases of minimal equilibration (massproportional heating) or full equilibration (all species rapidly heated to the same temperature). ${ }^{1}$ If cosmic ray production is included, the case considered is usually one with an extremely high injection efficiency of a thermal seed population into the cosmic ray acceleration process so that the cosmic ray flux at Earth can be explained. For observations that only measure one or two populations, the choice of equilibration can drastically affect the inferred age, explosion energy or distance. Furthermore, if equilibration is a function of shock speed, then even spanning both scenarios may be misleading because the evolution of the initial shock equilibration will effect the dynamics of the remnant (for instance consider the internal pressure). Calibrating a relationship between shock velocity and the efficiency of heating different particles to thermal and cosmic ray energies would be a fantastic boon for SNR studies if a clean relationship exists.

There are three generic methods for determining the temperature of particles behind a shock: (1) bremsstrahlung continuum emission, (2) the thermal broadening of a line from a particular species, and (3) the flux ratios of lines of either a single element or between elements. Each of these methods has its own

1 Bocchino et al. (1999) turned this argument around to estimate a degree of equilibration based on given Sedov parameters for the Vela supernova remnant 
pitfalls. (1) Both thermal bremsstrahlung and synchrotron radiation (from a high energy population of electrons) produce continuum emission in the $\mathrm{X}$ rays, and their relative contributions are not always possible to disentangle. Furthermore the bremsstrahlung continuum in the X-rays samples emission from an electron population significantly downstream from the shock. Assuming the electrons and protons were out of equilibrium the temperatures will equilibrate downstream on the timescale of coulomb collisions. The initial electron temperature must then be inferred using the ionization state to calibrate the coulomb timescale post-shock. (2) The line broadening is a function not only of the temperature of that species but also the velocity structure of the shock if the shocked filament is not resolved. (3) The temperature dependence of ionization and excitation by electrons and protons can be used to diagnose $T_{\mathrm{e}}$ or $T_{\mathrm{p}}$ from the line flux ratios. The fluxes also depend on the ion fractions or relative abundances which must be assumed if they cannot be derived. We will examine many manifestations of these generic methods and problems as the observational evidence unfolds.

The body of this review is organized by supernova in order of increasing velocity to emphasize the necessity of measurements in multiple wave-bands to corroborate the degree of equilibration. Although different authors use different parameters to express the degree of equilibration, the ratio of the initial electron to proton temperatures at the shock front $\left(T_{\mathrm{e}} / T_{\mathrm{p}}\right)$ will be used throughout this review. These studies all concentrated on the equilibration at the outer blast wave of each remnant. Further complications must be considered with regard to the energetics of a reverse shock into the pure metal ejecta, where the energetics are not dominated by the protons, cold electrons are continually released by ionization, and turbulence may generate a high magnetic field.

\section{The Cygnus Loop and RCW 86}

The ratio of broad-to-narrow component flux in the $\mathrm{H} \alpha$ and $\mathrm{H} \beta$ lines was used by Ghavamian et al. (2001) to measure the electron-proton temperature ratio behind shocks in the Cygnus Loop and RCW 86. The theory behind the broad and narrow Balmer line emission was first laid out by Chevalier et al. (1980). Directly behind a collisionless shock there can exist a population of cold neutral ions that were not affected by the shock passage. These neutrals will emit $\mathrm{H} \alpha$ and $\mathrm{H} \beta$ Balmer lines if they are collisionally excited before being ionized. This produces a narrow component of emission, whose width reflects the temperature of the pre-shock gas. The cold neutrals can also charge exchange with the shock-heated protons resulting in a population of hot neutrals. A broad component to the Balmer lines arises from this hot neutral population, where the width of the broad line represents the temperature of the post-shock protons. 

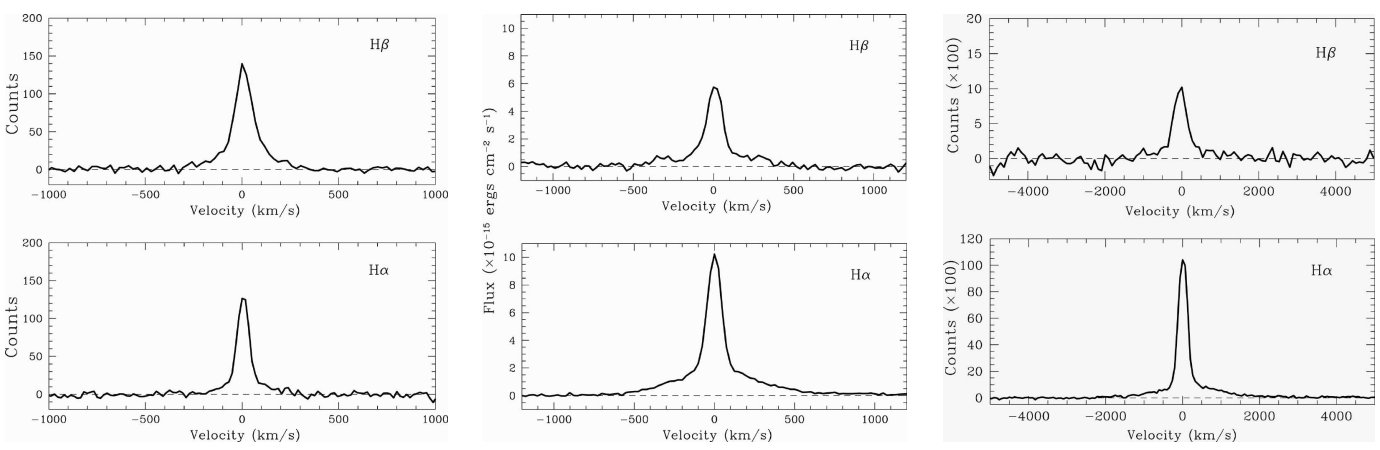

Fig. 1. The $\mathrm{H} \alpha$ and $\mathrm{H} \beta$ profiles of (a) the Cygnus Loop, (b) RCW 86 and (c) a compact knot in Tycho's SNR from Ghavamian et al. (2001). Note that the H $\alpha$ profile has higher signal to noise to constrain the broad-to-narrow ratio than does $\mathrm{H} \beta$, with the possible exception of the Cygnus Loop.

Figures 1a and 1b from Ghavamian et al. (2001) present the $\mathrm{H} \alpha$ and $\mathrm{H} \beta$ profiles from the Cygnus Loop and RCW 86. ${ }^{2}$ The post-shock proton temperatures derived from the widths of the $\mathrm{H} \alpha$ broad components are $T_{p}=(1.5 \pm$ $0.26) \times 10^{6} \mathrm{~K}$ for Cygnus and $T_{p}=(6.9 \pm 0.2) \times 10^{6} \mathrm{~K}$ for RCW 86. To estimate the degree of equilibration between electrons and protons Ghavamian et al. (2001) modeled the broad and narrow emission including the temperature dependence of charge exchange and excitation of the fast and slow neutrals by electrons and protons. The predicted broad-to-narrow ratios are plotted for different values of the initial neutral fraction in figures $2 \mathrm{a}$ and $2 \mathrm{~b}$ from Ghavamian et al. (2001). Using the $\mathrm{H} \beta$ ratio for Cygnus and the $\mathrm{H} \alpha$ ratio for RCW 86, they report constraints of $T_{\mathrm{e}} / T_{\mathrm{p}}=0.67-1.0$ and $T_{\mathrm{e}} / T_{\mathrm{p}}=0.25-0.43$, respectively. Assuming cosmic ray production was unimportant to the energetics of the shock, these equilibrations and the broad component widths imply shock speeds of 300-400 $\mathrm{km} \mathrm{s}^{-1}$ (Cygnus) and 580-660 $\mathrm{km} \mathrm{s}^{-1}$ (RCW 86).

However, it is worth noting from figure 2a that the $\mathrm{H} \alpha$ broad-to-narrow ratios predicted by Ghavamian et al. (2001) for the Cygnus loop lie 10\% higher than those observed. This level of discrepancy is readily explained by contamination of the narrow component by emission from a shock precursor. Some fraction of the hot neutrals will escape ahead of the shock before being ionized. Furthermore, in a cosmic-ray modified shock, some fraction of the cosmic rays will also escape into the pre-shock gas. The hot neutrals and cosmic ray particles will ionize and excite the medium ahead of the shock. This non-linear effect was not included in the models of Ghavamian et al. (2001) and in fact, modeling the balance between the production of hot neutrals and the continued existence of neutral atoms at the shock front is a work in progress (K. Korreck, personal communication). However, it is clear that precursor emission will increase the narrow component flux, shifting all the models to lower broad-to-narrow ra-

$\overline{2}$ The data are presented without the model so that the reader can better evaluate the reliability of the broad component measurement for each remnant in both $\mathrm{H} \alpha$ and $\mathrm{H} \beta$. 

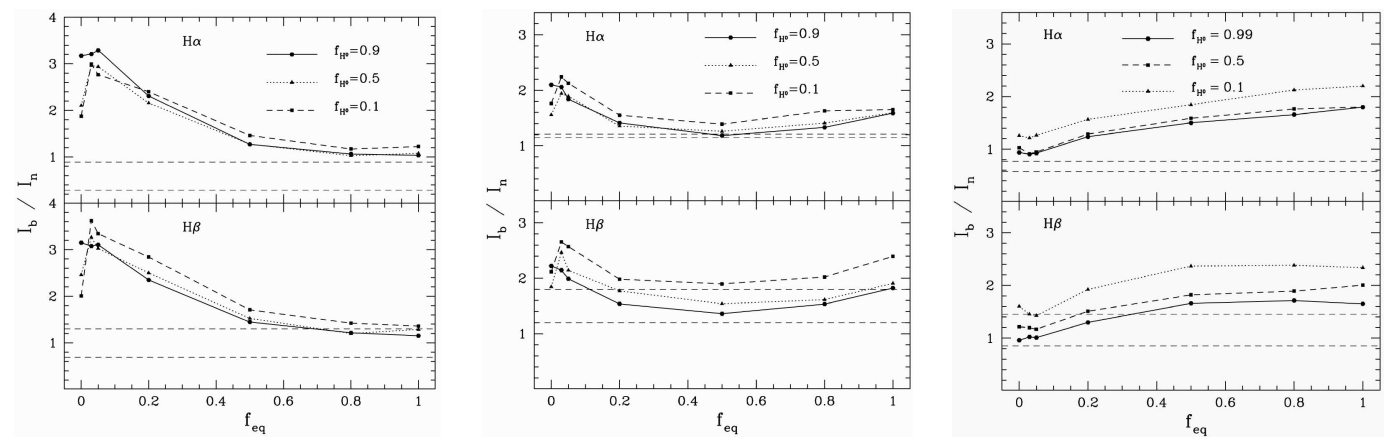

Fig. 2. The predicted $\mathrm{H} \alpha$ and $\mathrm{H} \beta$ broad-to-narrow ratios as a function of "fractional equilibration" from Ghavamian et al. (2001) for velocities appropriate for (a) the Cygnus Loop, (b) RCW 86 and (c) Tycho. Note that the model predictions are inconsistent with the higher signal-to-noise $\mathrm{H} \alpha$ broad-to-narrow ratio for the Cygnus Loop and Tycho's SNR.

tios. Given this uncertainty it is unclear whether a measured broad-to-narrow ratio lower than all of the models provides any constraint on $T_{\mathrm{e}} / T_{\mathrm{p}}$ at all. In fact in sections 3 and 4 we will see examples of shocks in which the broad-tonarrow ratio is significantly lower than any of Ghavamian's models, strongly indicating precursor emission. At present there is no reason to assume that this level of precursor contamination is missing in the Cygnus loop or RCW 86 just because their broad-to-narrow ratios happen to marginally intersect the predicted curves.

On the other hand, there is corroborating evidence for a high degree of equilibration in the Cygnus Loop. Ravmond et al. (2003) examined the far ultraviolet spectrum at the same position as was studied in Ghavamian et al. (2003). The width of the O IV $\lambda 1032$ line gives an upper limit on the oxygen temperature of $2.7 \times 10^{6} \mathrm{~K}$ (any velocity structure broadening would decrease this estimate). This is within a factor of 2.5 of the hydrogen temperature measured in Ghavamian et al. (2001). Given that mass-proportional heating would lead to a factor of 16 difference between the temperatures of oxygen and hydrogen, this measurement indicates a high degree of equilibration between ions at the shock. This lends credibility to the claim of high electron-ion equilibration. However, while the electron heating mechanisms may be tied to the temperature of the ions, there is still a three orders of magnitude difference in mass. Hence a high degree of equilibration between the ions themselves does not necessarily imply a similarly high degree of equilibration between the electrons and ions. 


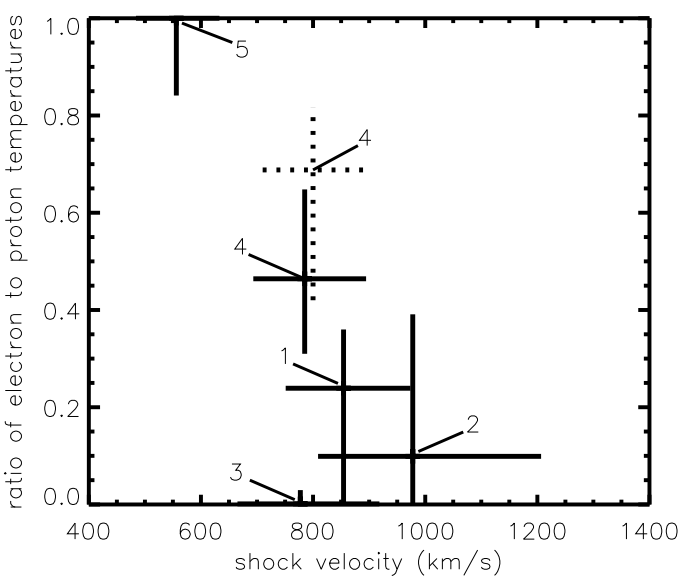

Fig. 3. Initial electron to proton temperature ratio as a function of shock speed for five apertures in DEM L71. Published results (Rakowski et al., 2003) are plotted as solid crosses, new result as dashed. The new analysis incorporates 100ks more data which enables constraints on the abundances for each region. The offset between the old and new results illustrates how an assumption about the underlying abundance set can bias the measured temperature.

\section{$3 \quad$ SNR DEM L71}

A combination of optical and X-ray observations was required to constrain the electron-proton temperature ratio around the blast-wave of SNR DEM L71 (Ghavamian et al., 2003; Rakowski et al., 2003). This Large Magellanic Cloud (LMC) remnant has many non-radiative $\mathrm{H} \alpha$ filaments around its outer rim, ranging in velocity from $\sim 500-1000 \mathrm{~km} \mathrm{~s}^{-1}$. Ghavamian et al. (2003) and Rakowski et al. (2003) chose apertures along the blast-wave to combine areas of similar optical properties into large enough regions for meaningful spectra in a $45.4 \mathrm{ks}$ Chandra observation. The optical measurements of the $\mathrm{H} \alpha$ broad component width (to obtain $T_{\mathrm{D}}$ ) and attempts to model the broad-to-narrow ratio are presented in Ghavamian et al. (2003). Without the addition of precursor emission the predicted broad-to-narrow ratios never drop below 1.0, but the measured flux ratios are never more than 0.74 (generally around 0.5). Until full precursor modeling is available no conclusion can be made about the degree of equilibration purely from the optical data.

In Rakowski et al. (2003), we extracted Chandra X-ray spectra from three nested regions behind the blast-wave at each aperture to measure the evolution of the electron temperature. Our final analysis of the initial ratio, $T_{\mathrm{e}} / T_{\mathrm{p}}$, utilized a planar shock model with the initial proton temperature fixed to the values from Ghavamian et al. (2003). The electron temperature as a function of distance behind the shock could then be parameterized in terms of the initial degree of equilibration. The published results are shown as solid crosses in figure 3. Except for the slowest shock (region 5), all of the shocks were consistent 
with no equilibration in the preliminary tests. Combining X-ray and optical results allowed us to identify one shock (region 4) with $T_{\mathrm{e}} / T_{\mathrm{p}} \sim 1 / 2$ whose spectrum was inconsistent with either full or minimal equilibration given the measured proton temperature. Limited statistics dictated that the abundances and column density be held constant in the X-ray fits. The chosen average abundance set will introduce a bias given the inverse correlation between the abundance and temperature when both are allowed to vary. The errors shown include an estimate of this systematic error as well as the measurement uncertainty in $T_{\mathrm{p}}$. An additional $100 \mathrm{ks}$ of Chandra observing time has allowed us to eliminate this systematic uncertainty by measuring the abundances and column density at each aperture. These data confirm that our shock model is sound, i.e. the temperatures and ionization timescales of the nested regions do indeed evolve when fit separately. The first of the updated measurements of $T_{\mathrm{e}} / T_{\mathrm{p}}$ is shown by the dashed cross in figure 3 confirming an intermediate degree of equilibration for region 4 . While the uncertainty remains similar, the result itself is no longer biased by an arbitrary choice of abundances.

\section{Tycho's SNR}

$\mathrm{H} \alpha$ and $\mathrm{H} \beta$ emission from a compact knot in Tycho's SNR were also included in Ghavamian et al. (2001) (see section 2). The spectra and model predictions for the broad-to-narrow ratios are shown here in figures $1 \mathrm{c}$ and $2 \mathrm{c}$ from Ghavamian et al. (2001). The $\mathrm{H} \alpha$ profile has considerably better signal to noise than $\mathrm{H} \beta$, but the $\mathrm{H} \alpha$ broad-to-narrow ratio falls below predictions even after accounting for some diffuse emission above the knot. Ghavamian et al. (2001) therefore use the less well-constrained $\mathrm{H} \beta$ ratio to estimate $T_{\mathrm{e}} / T_{\mathrm{p}}<$ 0.1. The predicted broad-to-narrow ratios in figure $2 \mathrm{c}$ certainly favor a low equilibration. The narrow component contribution from the cosmic-ray precursor would have to be more than one third of the total narrow $\mathrm{H} \alpha$ emission in order to be consistent with a $T_{\mathrm{e}} / T_{\mathrm{p}}$ ratio greater than 0.2 . However, a more precise statement on $T_{\mathrm{e}} / T_{\mathrm{p}}$ awaits a full precursor model to understand the difference between the predicted and observed $\mathrm{H} \alpha$ ratio.

Hwang et al. (2002) studied the Tycho SNR blast-wave with Chandra. Spectra extracted from narrow regions along the outer rim exhibited little or no line emission. Assuming this emission is thermal in origin a very young ionization age is needed to explain the lack of line emission given a fitted electron temperature around $2 \mathrm{keV}$. Given the low timescale, the electron temperature they measure should not have evolved since the initial shock heating. This electron temperature can be compared with a shock velocity derived (at a distance of $2.3 \mathrm{kpc})$ from radial expansion measurements in the radio $\left(3000-4000 \mathrm{~km} \mathrm{~s}^{-1}\right.$ Revnoso et al., 1997) and X-rays (4600 $\pm 400 \mathrm{~km} \mathrm{~s}^{-1}$ Hughes, 2000) to derive the ratio of the electron to mean temperatures. There remains a debate over 
which expansion measurement is representative of the present shock speed, however the electron-to-mean temperature ratio would be 0.10-0.20 assuming the mean radio expansion or $0.054-0.10$ for the X-ray expansion speed. Combining both these ranges yields a limit on $T_{e} / T_{\mathrm{D}}$ of $0.03-0.12$. This is in good agreement with the results of Ghavamian et al. (2001), however at a much faster shock within the same remnant. Hwang et al. (2002) note that the low measured electron temperature does not require a significant cosmic ray population to explain the gap between the mean temperature for the measured shock speed and the electron temperature. However, neither does it exclude the possibility that a significant fraction of the shock energy has gone into accelerating cosmic rays.

The above conclusions were derived under the assumption that the blast-wave emission is thermal. However, synchrotron emission arising from high energy electrons accelerated to cosmic-ray energies at the shock front is an equally natural explanation for such a featureless spectrum. The presence of hard Xray emission up to $30 \mathrm{keV}$ requires that a substantial portion of the $0.5-10$ $\mathrm{keV}$ continuum be nonthermal. (see section 4.2 of Hwang et al. (2002) for a full discussion) In fact, Hwang et al. (2002) were able to successfully model the line-free rim spectra with a synchrotron emission model that required consistency with radio measurements. They found that if the emission is primarily non-thermal, any additional thermal component would have to have a considerably lower electron temperature than was found assuming a completely thermal origin. This would be consistent with a picture where a large portion of the shock energy has gone into accelerating cosmic rays, leaving behind a low temperature population of thermal electrons, which may or may not be equilibrated with the thermal population of ions.

\section{SN 1006, fully unequilibrated shocks}

SN 1006 is the one remnant where the equilibration between particles has been truly well-studied. Four lines of evidence all indicate a low degree of equilibration or nearly mass-proportional heating. Any one estimate of $T_{\mathrm{e}} / T_{\mathrm{p}}$ may not be convincing, either because of its indirectness (Ravmond et al., 1995; Vink et al., 2003) or its dependence on arguments regarding the abundances or ion fractions (Ghavamian et al., 2002; Laming et al., 1996). However, the convergence of four largely independent measurements is compelling.

Ravmond et al. (1995) observed the ultraviolet spectrum from a 197" long region along the north-west shock measuring emission lines from $\mathrm{H}$ I, He II, C IV, $\mathrm{N} \mathrm{v}$, and $\mathrm{O}$ VI. They found that lines from all species show similar widths consistent with a speed of $2300 \mathrm{~km} \mathrm{~s}^{-1}$ indicating that ion-ion equilibration is ineffective. In Laming et al. (1996), they modeled the line ratios for this 
ultraviolet spectrum. From the ratio of two lines, one excited primarily by electrons, He II $\lambda 1640$, and one excited by protons, C IV $\lambda 1550$, one can infer the ratio of electron to ion temperatures. Assuming solar abundances and a factor of two carbon depletion onto grains, this flux ratio indicates $T_{\mathrm{e}} / T_{\text {ion }}<$ 0.05. However, since the flux ratio is obviously dependent on the assumed relative abundances, the authors admit that a ratio of $T_{\mathrm{e}} / T_{\mathrm{ion}}=0.2$ cannot be ruled out.

Ghavamian et al. (2002) re-examined the optical spectrum of the northwestern shock. The most useful diagnostic pair of lines were He I $\lambda 6678$ and He II $\lambda 4686$. The ratio of these two lines depends on the initial neutral fraction and the ratio of the He I excitation to ionization rates. The excitation to ionization rate drops sharply above $T_{\mathrm{e}}>10^{5} \mathrm{~K}$, implying that a low electron temperature is needed to produce measurable He I emission. A high neutral fraction is also necessary to replenish the supply of He I which is still ionizing faster than it emits photons. Quantitatively, comparing the electron temperature derived from modeling the He I to He II ratio with the proton temperature measured with the $\mathrm{H} \alpha$ broad component width, yields a limit of $T_{\mathrm{e}} / T_{\mathrm{p}}<0.03$. The other measured line ratios, $\mathrm{H} \mathrm{I} / \mathrm{H} \alpha$ and the broad-to-narrow ratios of $\mathrm{H} \alpha$ and $\mathrm{H} \beta$, while less directly diagnostic, are all consistent with a low equilibration, $T_{\mathrm{e}} / T_{\mathrm{p}}<0.07$.

Vink et al. (2003) studied the X-ray grating spectrum of a compact knot in the northwest using $X M M$-Newton. The broadening of a complex of $\mathrm{O} \mathrm{v}, \mathrm{O}$ VI and O VII lines imply an oxygen temperature of $k T_{\text {oxvoen }}=530 \pm 150 \mathrm{keV}$. If there has been no ion equilibration, as suggested by Ravmond et al. (1995), this oxygen temperature translates into a shock velocity of $v_{s}>4000 \mathrm{~km}$ $\mathrm{s}^{-1}$. If ion equilibration has occurred that would require an even faster, more energetic shock. Vink et al. (2003) use a CCD-resolution X-ray spectrum, extracted from the same $X M M-N$ ewton observation, to measure an electron temperature of $k T_{\mathrm{e}} \sim 1.5 \mathrm{keV}$. The ratio of the electron and oxygen temperatures imply an electron-to-proton temperature ratio of $\sim 0.05$ for the case of no ion equilibration, or even smaller values for a shock where the ions are equilibrated.

All of the measurements to date of SN 1006 indicate that the electrons and ions are far from being in temperature equilibrium. However, one of the most remarkable things about SN 1006 is the incomplete shell of bright radio and $\mathrm{X}$-ray synchrotron emission in the northeast and southwest indicating sites of efficient cosmic ray acceleration. All measurements concerning equilibration have been made for the northwestern rim, which does not seem to emit X-ray synchrotron emission. The situation for the synchrotron rims may be quite different. 


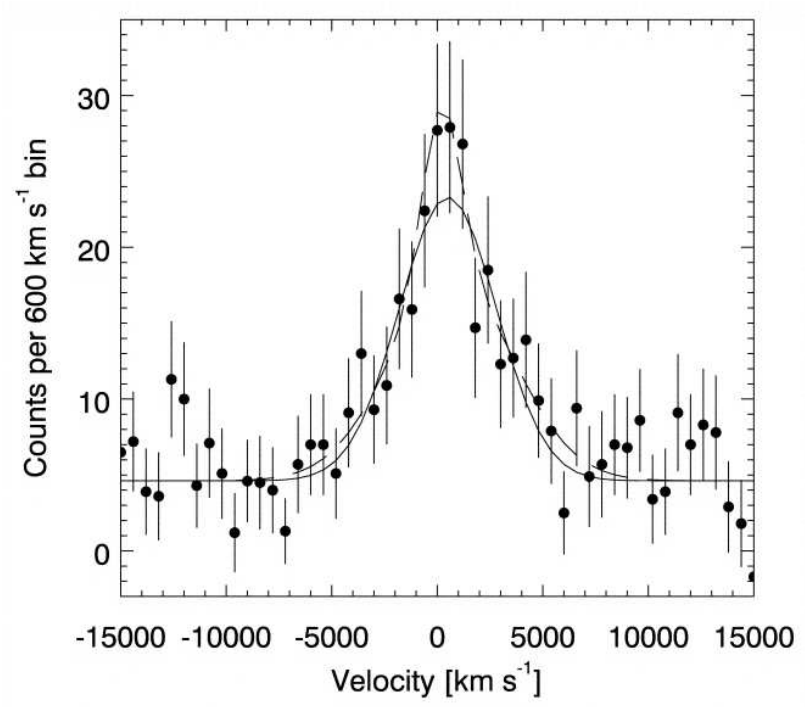

Fig. 4. Combined X-ray line profile from the grating spectrum of SN 1987A, reproduced from Michael et al. (2002) figure 8. Single (solid) and double (dashed) Gaussian fits are over-plotted.

\section{SN 1987A}

A single Chandra grating spectrum from an unresolved SNR, such as SN 1987A has the potential to measure the ion-ion and electron-ion equilibration simultaneously. The line ratios of individual elements can be used to constrain the electron temperature, while the line widths are a function of the temperature of that element and the profile of its bulk motion. The Chandra high energy transmission grating (HETG) spectrum of SN 1987A, presented by Michael et al. (2002), displays $\mathrm{K} \alpha$ and $\mathrm{L} \alpha$ lines of $\mathrm{N}, \mathrm{O}, \mathrm{Ne}, \mathrm{Mg}$, and Si. Constraints on $k T_{\mathrm{e}}$ and the ionization timescale from line ratios of individual elements were shown in Michael et al. (2002). Unfortunately the grating spectrum is not deep enough to constrain the temperature and timescale fully, however the line ratios agree with CCD spectrum best-fit values of $k T_{\mathrm{e}} \sim 2.6 \mathrm{keV}$ and $n_{\mathrm{e}} t \sim 6 \times 10^{10} \mathrm{~cm}^{-3} \mathrm{~s}$.

Ideally one would like to constrain each of the line widths separately, but again the statistics do not allow this. However, given that results from SN 1006 indicate a low degree of ion equilibration at such fast shocks, it is not unreasonable to assume that all the ions have the same velocity. Therefore, Michael et al. (2002) co-added the velocity profiles of the brightest lines of $\mathrm{N}$, $\mathrm{O}, \mathrm{Ne}, \mathrm{Mg}$, and Si to measure an "average" line-width. The combined X-ray line profile is reprinted here in figure 4. Since SN1987A is not resolved by the grating, this line profile includes both thermal velocity and bulk motion. Figure 5 (Michael et al., 2002) illustrates the line profiles produced by different geometries of the X-ray emitting plasma. Full equilibration between the ions produces sharper line profiles than no equilibration because of the greater bulk 

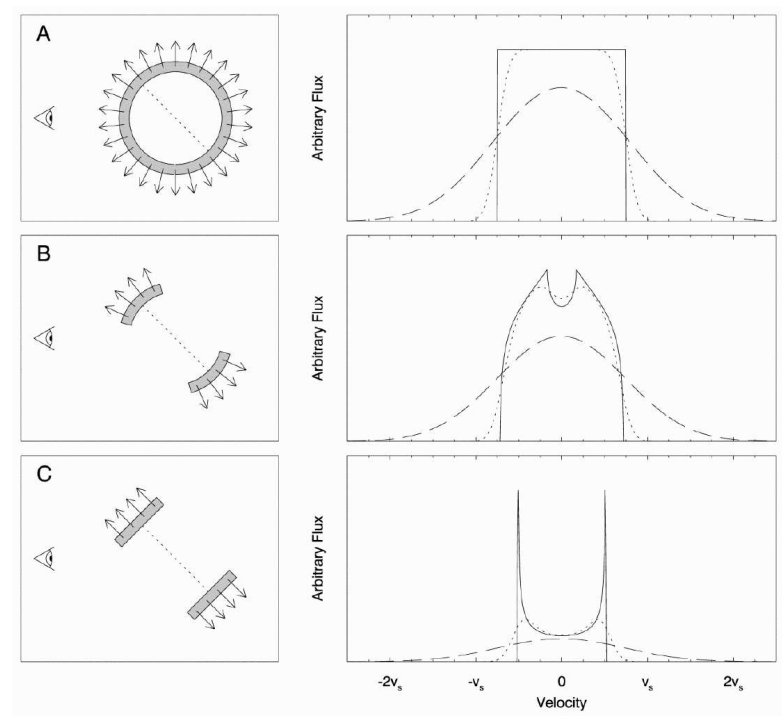

Fig. 5. Line profiles predicted for different geometries and degrees of equilibration for SN 1987A, reproduced from figure 9 of Michael et al. (2002). Solid lines show the bulk motion profiles. The dotted lines include the thermal broadening assuming full ion-ion equilibration. The dashed lines assume minimal equilibration at the shock front.

motion contribution. The smooth, no equilibration cases are more similar to the line profile seen. Michael et al. (2002) note that equatorial geometries are favored by other observations. For no ion equilibration and equatorial geometries $v_{s}=3400 \pm 700 \mathrm{~km} \mathrm{~s}^{-1}$, consistent with radio expansion measurements.

Similarly to what was done in DEM L71 by Rakowski et al. (2003), the velocity of the shock in SN 1987A can be used to constrain the CCD spectrum in terms of the initial electron to ion temperature ratio. Michael et al. (2002) find that if $v_{s} \sim 3500 \mathrm{~km} \mathrm{~s}^{-1}$, then the CCD spectrum is fit best by $T_{\mathrm{e}} / T_{s}=0.11 \pm 0.02$ (where $T_{s}$ is the average shock temperature, equivalently $T_{\mathrm{e}} / T_{\mathrm{p}}=0.07 \pm 0.01$ ). Unlike Rakowski et al. (2003), these constraints assume a fixed velocity and do not account for the range in velocities allowed by their line width calculations. However, the authors do note that fits with a very low electron temperature are inconsistent with the data, thus excluding the case of zero election-ion equilibration.

\section{$7 \quad 1 \mathrm{E} 0102-72$}

Up until this section, all discussions have assumed that the acceleration of cosmic rays is not an important factor in the shock dynamics. However it is widely believed that SNR shocks are the main source of the bulk of the cosmic ray distribution. If cosmic ray acceleration is efficient a significant portion of the shock energy may be diverted from the thermal populations. This should 
have important consequences on the shock itself (Decourcelle et al., 2000). The shock compression will rise due to the lower adiabatic index of relativistic particles, the forward and reverse shocks will be closer together and the temperature and density gradients will be more severe. As cosmic rays escape the shock they carry away shock energy, making these dynamic effects more dramatic. Neglecting the energetics of cosmic ray acceleration when considering the equililbration of the thermal populations can give misleading results. If cosmic ray acceleration is important then results that are based on an expansion velocity and an electron temperature may in fact have a much higher degree of equilibration between the thermal populations (i.e. the Tycho results of Hwang et al., 2002). Furthermore, inferring a velocity purely from the electron and ion temperatures will underestimate the shock speed if much of the shock energy is in relativistic particles.

Hughes et al. (2000) used Chandra calibration observations of 1E 0102-72 to measure the expansion of the SNR since the earlier Einstein and ROSAT images. With the known $50 \mathrm{kpc}$ distance to the LMC, the $0.1 \%$ expansion per year implies a $\sim 6000 \mathrm{~km} \mathrm{~s}^{-1}$ shock speed for the outer blast-wave. An X-ray spectrum of the blast-wave, extracted from the same observation, exhibits an electron temperature of less than $1 \mathrm{keV}, 25$ times smaller than the minimum average temperature implied by the measured shock velocity. There is no initial $T_{\mathrm{e}} / T_{\mathrm{p}}$ ratio for which the gulf between this average temperature and the electron temperature can be explained. Even after accounting for adiabatic decompression and only coulomb heating post-shock (up to the ionization timescale seen in the spectrum) the minimum electron temperature for such a fast shock is no lower than $\sim 2.5 \mathrm{keV}$ for the extreme case of mass-proportional heating. The discrepancy between the low electron temperature and high energy of the shock requires the existence of another population into which the shock energy is flowing. If a significant fraction of the shock energy has gone into accelerating cosmic rays then our comparison of the electron temperature with the shock velocity tells us nothing about $T_{\mathrm{e}} / T_{\mathrm{p}}$. In fact, non-linear models of shock acceleration (Ellison, 2000), at Mach numbers appropriate for 1E 0102-72, exhibit electron temperatures near $1 \mathrm{keV}$ and fully equilibrated electron and proton thermal populations.

\section{Conclusions}

The preceding results are compiled in figure 6 . Note the new constraint for region 4 in DEM L71, obtained using a Chandra observation three times as long as the original. This region still requires an intermediate degree of equilibration even without assuming a particular abundance set. Recall also that corroborating ion-ion equilibration measurements exist for SN 1006 (Ravmond et al., 1995, mass-proportional heating) and the Cygnus Loop (Ravmond et al., 2003, 


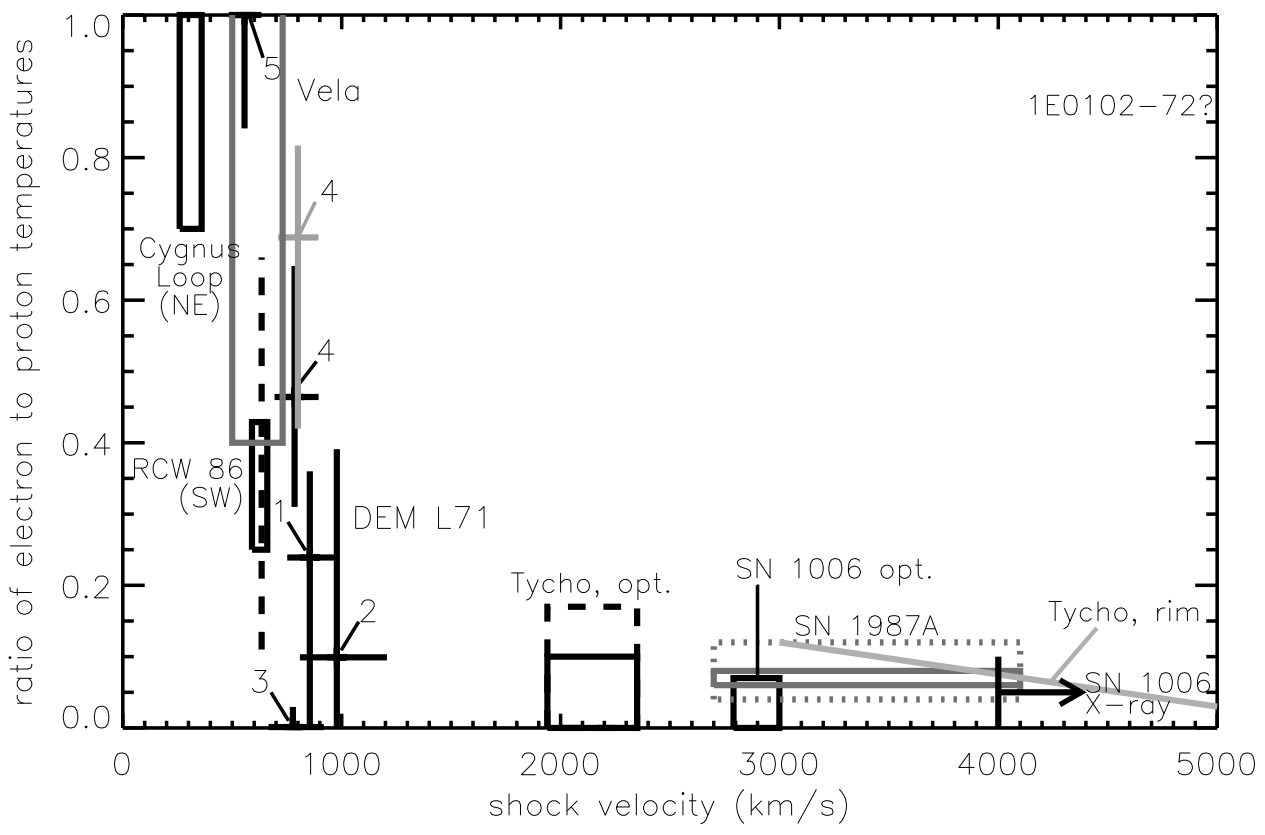

Fig. 6. Compilation of current electron-ion equilibration measurements at SNR shocks. The solid boxes for the Cygnus Loop, RCW 86 and Tycho, are from Ghavamian et al. (2001) modeling the broad-to-narrow ratios of $\mathrm{H} \alpha$ and $\mathrm{H} \beta$. The dashed lines are my estimate using only the $\mathrm{H} \beta$ measurement, however this estimate does not include the potentially significant effect of precursor contamination to the narrow component. The grey box marked "Vela" shows the estimate by Bocchino et al. (1999) of the current $T_{\mathrm{e}} / T_{\mathrm{p}}$, which did not take into account the amount of coulomb equilibration that has already occurred. Crosses show DEM L71 results from Rakowski et al. (2003) using the broad $\mathrm{H} \alpha$ component to fix the proton temperature in an X-ray fit of $T_{\mathrm{e}} / T_{\mathrm{p}}$. The grey cross indicates the updated result from deeper observations which allow us to simultaneously constrain the elemental abundances rather than fixing them to some average abundance set. The two SN 1006 results indicate the most constraining of the optical or UV line ratio measurements (Ghavamian et al., 2003), and the X-ray grating measurement of the oxygen line width and electron temperature (Vink et al., 2003). The solid box for SN 1987A shows the reported results from Michael et al. (2002) using a fixed shock velocity to constrain $T_{\mathrm{e}} / T_{\mathrm{p}}$ from the X-ray CCD spectrum, the dashed box gives an estimate which includes the error in their measurement of the shock velocity. The Hwang et al. (2002) measurement plotted here, converted from their electron-to-mean temperature ratio for Tycho's outer rim, compares the electron temperature measurement with both the radio and X-ray expansion velocities. For 1E $0102-72$, the low electron temperature could not be explained by a minimal degree of electron-ion equilibration at the shock front but rather required that a significant portion of the shock energy be going into the acceleration of cosmic rays. If the injection efficiency into the acceleration process is high, models predict that the remaining thermal populations may be equilibrated. 
$T_{\text {oxugen }}<2.5 \times T_{\mathrm{p}}$ ). Also included in this figure is the report by Bocchino et al. (1999) of $T_{\mathrm{e}} / T_{\mathrm{p}}$ between 0.4 and 1 for an approximately $600 \mathrm{~km} \mathrm{~s}^{-1}$ shock in the Vela SNR using ROSAT PSPC spectra. This was estimated by comparing the electron temperature of the hotter of two collisional ionization equilibrium components with a proton temperature derived from their best estimates for the Sedov parameters for Vela. However a measurement of the ionization timescale is needed to calibrate the extent to which coulomb collisions have already equilibrated the electron and proton temperatures as was done for DEM L71 and 1E 0102-72.

An inverse correlation between shock velocity and efficiency of electron heating has been suggested based on different subsets of these results. However, even if we accept that the high equilibration result for the Cygnus Loop is not an artifact of neglecting the pre-cursor emission, we are still left with one secure identification of an intermediate equilibration in the interesting range from 400 to $2000 \mathrm{~km} \mathrm{~s}^{-1}$ where the transition from near full equilibration to low equilibration should occur. Furthermore, once cosmic ray acceleration is considered, the shock velocities that were inferred from thermal line-widths may be grossly underestimated. Additionally, the fast shocks in Tycho, while not requiring a large cosmic ray population, do not exclude it either. If the outer blast-wave of Tycho is efficiently accelerating particles to cosmic ray energies, synchrotron radiation from high energy electrons may explain the featureless rim spectra while the thermal populations could be nearly equilibrated similarly to $1 \mathrm{E} 0102-72$.

Progress in this developing field depends on accurate modeling of the emission from pre-shock and post-shock gas, corroborating evidence from multiple wave-bands, and useful assessments of the cosmic ray production and its effect on the shock. The spectral resolution of future X-ray missions such as ASTRO-E2, ConX and Xeus will allow thermal Doppler broadening to be measured routinely in the X-rays as they now are in the optical and ultraviolet. Observations of 500-2000 $\mathrm{km} \mathrm{s}^{-1}$ shocks, where the transition from full to minimal equilibration should occur are especially important for diagnosing the relationship between shock velocity and the relative importance of different collisionless heating processes.

I would like to thank Arend Sluis, Parviz Ghavamian and Kelly Korreck for fruitful discussions regarding this review and shock physics in general. This review also benefited from the comments of two conscientious referees. This work was supported by NASA grant NAG5-9281. 


\section{References}

Bocchino, F., Maggio, A., and Sciortino, S. "ROSAT PSPC observation of the NE region of the Vela supernova remnant III. The two-component nature of the X-ray emission and its implications on the ISM" Astron. Astrophys. 342, 839-853, 1999.

Chevalier, Roger. A., Kirshner, Robert. P., and Raymond, John. C. "The optical emission from a fast shock wave with application to supernova remnants". ApJ 235, 186-195, 1980.

Decourchelle, Anne, Ellison, D. C., and Ballet, Jean "Thermal X-Ray Emission and Cosmic-Ray Production in Young Supernova Remnants" ApJ 543, L57L60, 2000.

Ellison, D. C. "The Cosmic Ray-X-ray Connection: Effects of Nonlinear Shock Acceleration on Photon Production in SNRs" in AIP Conf. Proc. 528, Acceleration and Transport of Energetic Particles Observed in the Heliosphere. Edited by Richard A. Mewaldt, J. R. Jokipii, Martin A. Lee, Eberhard Möbius, and Thomas H. Zurbuchen. Melville, (New York: AIP), pp. 386-93, 2000.

Ghavamian, Parviz, Raymond, John C., Smith, R. Chris, and Hartigan, Patrick "Balmer-dominated spectra of nonradiative shocks in the Cygnus Loop, RCW 86, and Tycho supernova remnants." ApJ 547, 995-1009, 2001.

Ghavamian, Parviz, Winkler, P. Frank, Raymond, John C., and Long, Knox S. "The optical spectrum of the SN 1006 supernova remnant revisited." ApJ, 572, 888-896, 2002.

Ghavamian, Parviz, Rakowski, Cara E., and Hughes, John P. "The physics of supernova remnant blast waves. I. Kinematics of DEM L71 in the Large Magellanic Cloud." ApJ 590, 833-845, 2003.

Hughes, John P. "The Expansion of the X-ray Remnant of Tycho's Supernova (SN 1572)" ApJ, 545, L53-L56, 2000.

Hughes, John P., Rakowski, Cara E., Decourchelle, Anne "Electron heating and cosmic rays at a supernova shock from Chandra X-ray observations of 1E 0102.2-7219." ApJ 543, L61-L65, 2000.

Hwang, Una, Decourchelle, Anne, Holt, Stephen, S., and Petre, Robert "Thermal and nonthermal X-ray emission from the forward shock in Tycho's supernova remnant." ApJ 581, 1101-1115, 2002.

Laming, J. Martin, Raymond, John C., McLaughlin, Brendan M. and Blair, William P. "Electron-ion equilibration in nonradiative shocks associated with SN 1006." ApJ 472, 267-274, 1996.

Lembege, B., Giacalone, J., Scholer, M., et al. "Selected problems in collisionless-shock physics" Space Science Reviews 110, 161-226, 2004.

McKee, Christopher F., and Hollenbach, David J. "Interstellar Shock Waves" Ann. Rev. Astron. Astrophys. 18, 219-262, 1980.

Michael, Eli, Zhekov, Svetozar, McCray Richard, et al. "The X-ray spectrum of supernova remnant 1987A." ApJ 574, 166-178, 2002.

Rakowski, Cara E., Ghavamian, Parviz, and Hughes, John P. "The physics of 
supernova remnant blast waves. II. Electron-ion equilibration in DEM L71 in the Large Magellanic Cloud." ApJ 590, 846-857, 2003.

Raymond, John C., Blair, William P., and Long, Knox S. "Detection of ultraviolet emission lines in SN 1006 with the Hopkins Ultraviolet Telescope." ApJ 454, L31-L34, 1995.

Raymond, John C., Ghavamian, Parviz, Sanskrit, Ravi, et al. "Far-ultraviolet spectra of a nonradiative shock wave in the Cygnus Loop." ApJ 584, 770781, 2003.

Reynoso, E. M., Moffett, D. A., Goss, W. M., et al. "A VLA Study of the Expansion of Tycho's Supernova Remnant" ApJ 491, 816-828, 1997.

Schwartz, Steven. J., Thomsen Michelle. F., Bame, S. J., and Stansberry, John "Electron heating and the potential jump across fast mode shocks" J. Geophys. Rev. 93, 12923-12931, 1988.

Vink, Jacco, Laming, J. Martin, Go, Ming Feng, et al. "The slow temperature equilibration behind the shock front of SN 1006." ApJ 587, L31-L34, 2003. 